\title{
Coherent double neutral Pion Photoproduction off Deuterons
}

\author{
Michael Sven Guenther* \\ For the A2 Collaboration \\ Departement of Physics, University of Basel \\ E-mail: m.guenthereunibas.ch
}

\begin{abstract}
Photoproduction of meson pairs off nucleons has gained a lot of interest because it allows to study sequential decays of nucleon resonances via some intermediate excited states. This may give access to states that have only tiny decay branching ratios for direct decays to the nucleon ground state by emission of a single meson. In particular, $\pi$ pairs and $\pi \eta$ pairs have been studied in detail during the last few years. In the present talk, we discussed recent results from the production of $\pi^{0}$ pairs from deuterons. The study of quasi-free production from protons and neutrons bound in the deuteron helps to disentangle the isospin decomposition of the production amplitudes. However, for $\pi^{0}$ pairs, the coherent reaction mechanism off the deuteron, i.e. the final state $\pi^{0} \pi^{0} d$, is of great interest. Also this reaction, can contribute to the isospin decomposition, but there is a more exciting aspect. Recently, a narrow resonance structure has been observed in the $p n \rightarrow d \pi^{0} \pi^{0}$ reaction, which has been discussed as a possible candidate for an unconventional six-quark, the $d^{*}(2380)$ dibaryon resonance. If such a state exists, it should in principle also show up in $\gamma d \rightarrow d \pi^{0} \pi^{0}$, although the production cross section would be much smaller than in the hadron induced reaction. Coherent photoproduction of mesons off the deuteron or other light nucleons has so far not been extensively explored. The only final state which is reasonably well studied is $d \pi^{0}$ in the energy range of the $\Delta$ resonance. Apart from that, there are only few results for $\eta, \eta^{\prime}$, and $\eta \pi$ production. In the present contribution, we summarize recent preliminary results for the $\gamma d \rightarrow d \pi^{0} \pi^{0}$ reaction. The experiment was performed at the tagged photon beam of the MAMI accelerator in Mainz, Germany, with the combined Crystal Ball/TAPS electromagnetic calorimeter. The excitation function for this reaction was studied from the energy region of the tentative $\mathrm{d}^{*}(2380)$ state throughout the second and third resonance region of the nucleon.
\end{abstract}

XVII International Conference on Hadron Spectroscopy and Structure - Hadron2017

25-29 September, 2017

University of Salamanca, Salamanca, Spain

${ }^{*}$ Speaker. 


\section{Introduction}

The strong interaction, responsible for the binding of quark in nucleons, is of great interest, especially since the understanding of the lower energy region has not proved satisfactory. Besides the most famous missing resonance problem, many other conflicts between theory and experimental results occurred during the study of the interaction.

Photoproduction of mesons has become one of the most important tools for studying the strong interaction responisble for the excites states of nucleons. In this region, the strong interaction can no longer be calculated using perturbation theories, instead lattice gauge theories are used to describe the excited stated of nucleons. To further improve the understanding of the strong interaction in the lower energy region, the detailed study of these nuclear resonances is necessary. Photoproduction of meson pairs off nucleons allows for the study of sequential decays of such resonances via some intermediate excited states. With the growing accuracy of the experiments, we can now study reactions with small production rates. In particular, $\pi$ pairs and $\pi \eta$ pairs have been studied in more detail during the last few years[2].

In this paper, preliminary results from the production of $\pi^{0}$ pairs of deuterons will be presented. This production channel should theoretically offer an access to the $d^{*}(2380)$ dibaryon resonance. The excitation function for the coherent photoproduction of $\pi^{0}$ pairs of deuterons was studied from reaction threshold throughout the second and third resonance region of the nucleon.

\section{Experimental Settings}

The data presented was taken by the A2-Collaboration, with a combination of the Crystal Ball/TAPS detector setup and the MAMI electron accelerator in Mainz, Germany. MAMI is an electron accelerator, built as a cascade of racetrack microtrons (RTMs), with its final stage, the Harmonic Double Sided Mircrotron (HDSM), reaching electron energies up to $1.6 \mathrm{GeV}$. The electron beam impinged on a radiator foil and produced the photon beam via bremsstrahlung. The produced beam of photons is, depending on the running conditions, unpolarized or circuluary or linearly polarized. The photon beam irradiates the production targets, for which different materials (hydrogen, deuterium, helium, heavier nuclei) can be used, some of them also polarized (frozenspin buthanol targets)[4].

The main detector is a combination of the Crystal Ball (CB) and the Two Armed Photon Spectrometer (TAPS). The CB is a nearly $2 \pi$ spectrometer where only the entrance and exit windows of the photom beam $\left(20^{\circ}\right)$ are not covered. The exit window in forward direction is covered by the TAPS forward wall down to $5^{\circ}$. In combination with CB/TAPS, two particle identification detectors (PID inside the CB around the target and VETO in front of TAPS) are installed. This overall setting provides a high-precision measurement of mesons by detecting the corresponding decay photons with high efficiency [4]. 


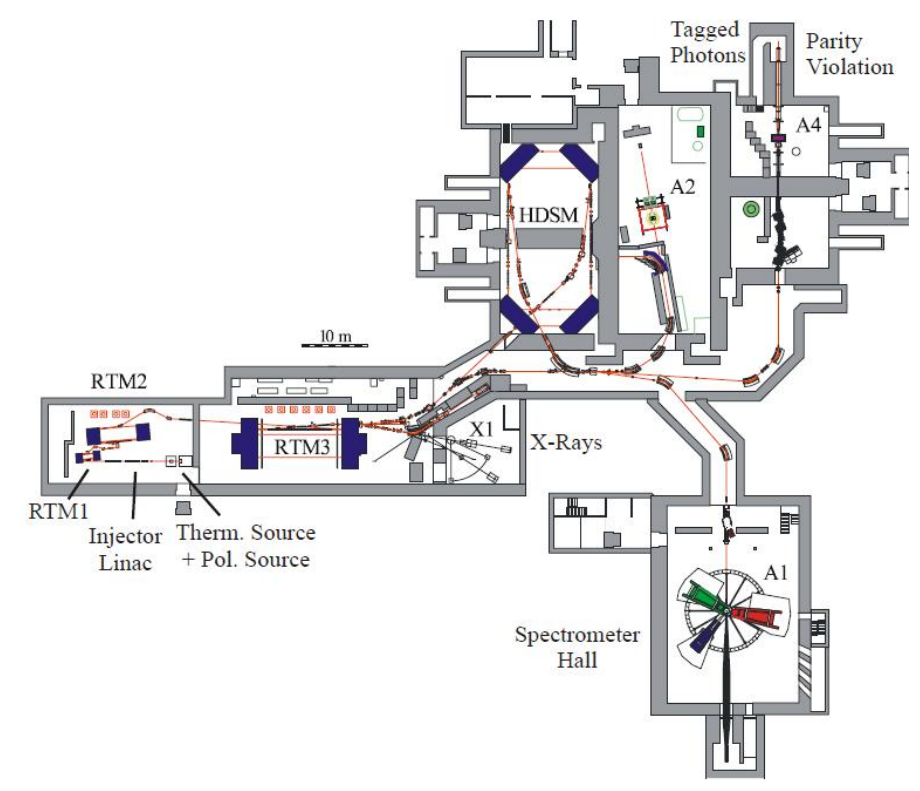

Figure 1: The MAinzer MIcrotron electron accelerator facility. Figure taken from [3]

\section{Analysis}

The data set was searched for events with four neutral hits and one charged hit. The latter was handled as the deuteron candidate, while the four neutral hits were handled as the decay photons coming from the $\pi^{0}$ s. A $\chi^{2}$-search algorithm was used to find the best combination for building the $\pi^{0}$ pair out of the four photons. In order to identify the coherent reaction channel, several kinematic cuts were applied. These include cuts on the $\theta$ and $\phi$ angular distribution, the missing mass, invariant mass, and a time of flight analysis. The latter one is crucial to distinguish between deuterons and protons, which are coming from the quasi-free reaction, the main background for this reaction. The time of flight spectra can be seen in figure 2. For a final cross check, the missing mass spectrum of all unfiltered events is analyzed in order to determine a signal to background ratio.

The detection efficiency, needed for the calculation of the total cross section, was determined using a Monte Carlo simulation with Geant 4 . The cross section was calculated using:

$$
\sigma_{t o t}\left(E_{\gamma}\right)=\frac{N_{g}\left(E_{\gamma}\right)}{\Phi\left(E_{\gamma}\right) \varepsilon\left(E_{\gamma}\right) \Gamma_{b} \rho_{T}},
$$

where $N_{g}$ is the number of good events getting through the analysis filter, $\Phi$ is the incoming flux of photons, $\varepsilon$ is the tagging effinciency, $\Gamma_{b}$ is the branching ratio of the $\pi^{0}$ decay into two photons, which is $\approx 98,82 \%$ [5], and $\rho$ is the target density. 


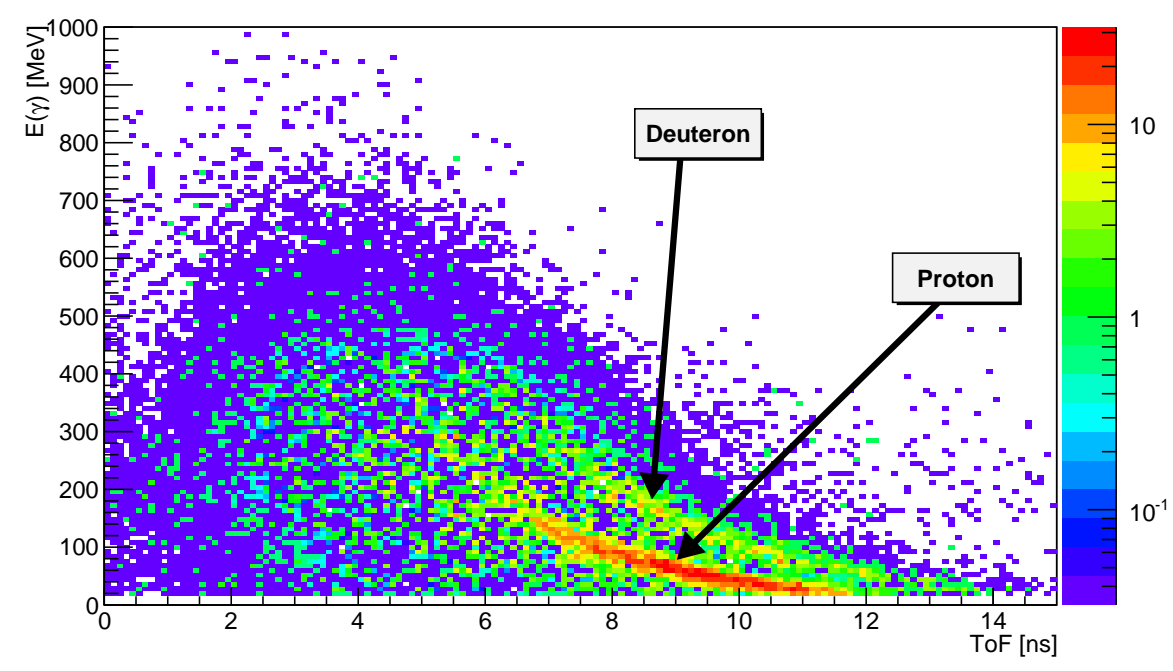

Figure 2: Time of flight spectrum, which shows the possibility of distinguish between proton and deuteron within the analysis software.

\section{Preliminary Results}

The preliminary results for the coherent double $\pi^{0}$ photoproduction are shown in Figure 2. The total cross section is in the range of $0.08 \mu \mathrm{barn}$, which is orders of magnitude lower than the quasi-free production on the deuteron. However, this is to be expected. For comparison, results from a model [1] published by A. Fix et al. are shown in figure 3. After the first rise of the cross section, a double bump structure can be seen. It is yet to be determined how the signal from the proposed di-baryon resonance would influence the cross section in the $W$ range where an enhancement of the experimental data over the results from the model of Fix et al. is observed. The cross section is presented dependent on $W . W$ represents the invariant mass of the combined photondeuteron-system. In this figure, only the statistical errors are shown.

Additionally, also coherent triple $\pi^{0}$ production was analyzed. In the threshold region it is almost impossible to obtain results for the elementary $\gamma p \rightarrow p 3 \pi^{0}$ reaction because this is completely dominated by the $\eta \rightarrow 3 \pi^{0}$ decay. However, coherent production of $\eta$-mesons off the deuteron is strongly suppressed. The results are shown in Figure 4. The cross section is of similar magnitude as the double $\pi^{0}$ channel. The triple $\pi^{0}$ channel has a slightly higher production threshold which also is represented in the data. A dominant structure within the cross section can not be seen. It still need to be analyzed how big the influence from the coherent reaction: $\gamma d \rightarrow d \eta \rightarrow d 3 \pi^{0}$ for this data set is. Regarding these uncertainties the preliminary results look quite promising and a further analysis is already on its way.

Overall, a precise measurement for the coherent double and triple $\pi^{0}$ photoproduction cross section was achieved. However, it is mandatory to mention that both data sets are still under investigation and the results shown are preliminary. 


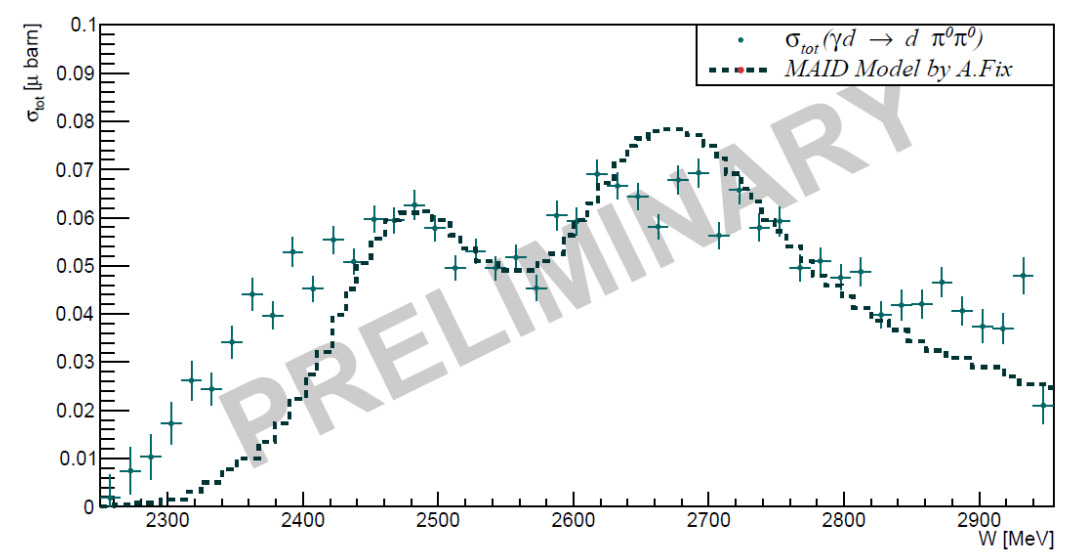

Figure 3: Total cross section for the reaction: $\gamma d \rightarrow d \pi^{0} \pi^{0}$. The production threshold is at around 2280 $\mathrm{MeV}$. Up until $2440 \mathrm{MeV}$, the cross section is higher than the model. The model does not include a possible dibaryon resonance. For energies higher than $2440 \mathrm{MeV}$, the prediction and the results are comparable. The double peak structure can mostly be recreated in the data. The maximum height is reached at $0.07 \mu$ barn at around $2700 \mathrm{MeV}$ within the second peak. After the second peak, a continuous decrease can be seen.

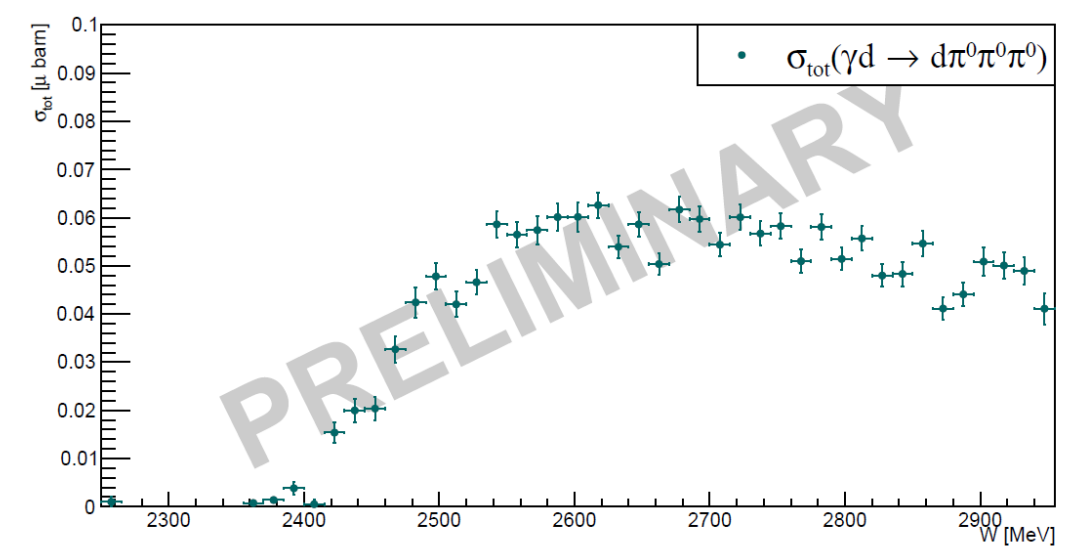

Figure 4: Total cross section for the reaction: $\gamma d \rightarrow d \pi^{0} \pi^{0} \pi^{0}$. The production threshold is at around 2400 $\mathrm{MeV}$. After the initial rise to the max height of $0.06 \mu$ barn, the cross section stays rather flat with a slow decrease.

\section{References}

[1] A. Fix et al.: Double pion photoproduction on nucleon and deuteron, Eur.Phys.J. A25 (2005) 115-135 [ arXiv:nucl-th/0503042]

[2] B. Krusche:Photoproduction of mesons of nucleons, Eur. Phys. J. Special Topics 198 (2011) 199 [ arXiv:1110.0192]

[3] Institut for nuclear physics Johannes Guttenberg Universitiy of Mainz: Floorplan of MAMI facility Mainz, 2006 
[4] D. Werthmueller: Experimental study of nucleon resonance contributions to eta-photoproduction on the neutron, PHD Thesis (2014)

[5] Particle Data Group: Review of particle physics, Chinese Physics C 38(9):090001 (2014) 